\title{
Liste des thèses d'anthropologie soutenues en 2010
}

\section{Université de la Méditerranée Aix-Marseille 2}

Isabelle BOUCHEZ : Evolution des marqueurs non conventionnels âge et sexe dépendants : apport de la paléopathologie

Aude CIVETTA : Les populations du Néolithique moyen autour du bassin lémanique : une approche anthropométrique

Philippe DE SOUTO BARRETO : Danse et vieillissement : Liens entre capacité physique fonctionnelle et indicateurs psychosociaux

Céline EMERIAU : Eclaircissement de la peau chez les femmes africaines à Marseille

Marilena GIROTTI : Etude biodémographique de deux populations des Alpes occidentales : Chiomonte (haute vallée de Susa - Italie) et l'Argentière - la-Bessée (Hautes Alpes - France)

Géraud GOURJON : Estimation du mélange génétique dans les populations humaines

Patrick HERVIEU : Gestion funéraire et palébiologie de sépultures collectives au Moyen Age et à l'Epoque Moderne : l'exemple des caveaux

Virginie MASDOUA : Communications et informations sur l'alimentation et la nutrition dites bénéfiques pour la santé. Les influences sur les savoirs et les pratiques corporelles d'adultes vivant en France contemporaine

Judith NICOGOSSIAN : De la reconstitution à l'augmentation du corps humain en médecine restaurative et en cybermétrique

Sandy PARMENTIER : Une nouvelle méthode d'estimation du Nombre Minimum d'Individus (NMI) par une approche allométrique. Applications aux séries ostéologiques de la région Provence-Alpes-Côte d'Azur

Michel RUQUET : Intérêts de la variabilité de l'alvéolyse humaine dans l'estimation de l'âge en anthropologie médico-légale

\section{Université Bordeaux 1}

Nathalie DELHOPITAL: Du monde des vivants au monde des morts en Nabatène, entre le $2^{e} s . A V$. J.-C. et le $4^{e} s$. A.P.J.-C. : approche archéo-anthropologique des tombes de Khirbet Edh-Dharih, Pétra (Jordanie) et de Madâ'in Saleh (Arabie-Saoudite)

Fabienne JORDANA : Fracturation sur os frais ou sur os sec de la voûte crânienne : approche méthodologique et application archéologique.

Joyce NASSAR : Les espaces funéraires suburbains de Mari (Moyen-Euphrate, 2900-1760 av. J.-C.) : analyse archéo-anthropologique

\section{Université Paris 6}

Laure SEGUREL : Influence du mode de vie sur la diversité génétique dans des populations humaines d'Asie Centrale

\section{Muséum national d'Histoire naturelle, Paris}

Aurélie DRUGUET : De l'invention des paysages à la construction des territoires : les terrasses des Ifugao (Philippines) et des Cévenols (France)

Erik GUSTAFSSON : Étude expérimentale ex et in situ de la sélection de plantes à propriétés thérapeutiques par les grands singes : influence de la physiologie, de l'écologie et de la structure sociale sur l'acquisition du comportement

Marine ROBILLARD : Pygmées Baka et voisins dans la tourmente des politiques environnementales en Afrique centrale

Beatriz SOENGAS : La subsistance des Pygmées Bakoya à l'épreuve de l'agriculture : dynamique des savoirs ethnobotaniques et des pratiques (Département de la Zadié, Ogooué-Ivindo, Gabon) 